\title{
Estimation of Melting of Moving Target Based on Background Subtraction
}

\author{
Gege Tian $^{a, *}$ \\ School of Software, Zhengzhou University, Zhengzhou, 450002, China \\ ${ }^{a}$ Con_pare@hotmail.com \\ ${ }^{*}$ Corresponding author
}

Keywords: Background subtraction; Pixel volume; Melting State.

\begin{abstract}
In this paper, the image processing technology is used to remove the interference factors of the original image, and then the time-series image is further processed based on the background difference method and centroid correlation method. The binary image containing only the target crystal can be extracted more accurately, and the two-dimensional centroid coordinates of the target crystal can be calculated. We use the idea of segmentation to divide the silicon dioxide region into several quasi-circular regions, calculate the volume of crystal pixel, estimate the actual volume of crystal according to the ratio of background reference object and image pixel, and then calculate the mass. Later, we get the melting rate through the derivation of mass to time. Finally, we use MATLAB to fit the volume and time of silica, when it is exponential function the fitting degree is the best. The model not only provides guidance for the recovery of iron tailings but also provides ideas for the establishment of a crystal tracking model and melting speed model for tracking melting in the molten pool.
\end{abstract}

\section{Introduction}

Iron tailing is the main solid waste discharged from the concentrator, and its recycling has always been a problem of great concern to the whole society. [1] One of the difficulties in recycling is how to grasp the opportunity of addition and heat compensation in the process of slag cotton preparation.

The melting of iron tailings can be characterized by the melting of silica. A new type of non-contact CCD shooting system is used to capture and track the molten silica in the molten pool, and then the real-time melting speed of silica can be obtained through the analysis of the shooting video. Therefore, it is of great significance for the recovery and utilization of iron tailings to analyze and process the silicon dioxide melting image and establish the silicon dioxide melting model based on the image analysis.

\section{Centroid calibration based on background subtraction method}

\subsection{Image processing based on background subtraction}

In the process of image acquisition, many noise signals will be superimposed on the image. At the same time, the unfixed silica particles are colorless and transparent, and the gray contrast with other parts of the crucible is weak. Therefore, to extract the contour and position information of silica, the image must be preprocessed by gray level processing, noise reduction, and filtering.

The first step of tracking the fused silica is to detect the moving target of silica particles in the sequential image. To extract the target easily and accurately, we further processed the image based on background subtraction [4]. The main idea of the background difference method is to extract the target region by differential operation between the current frame image and a constantly updated background model[2].The flow chart in Fig.1. 


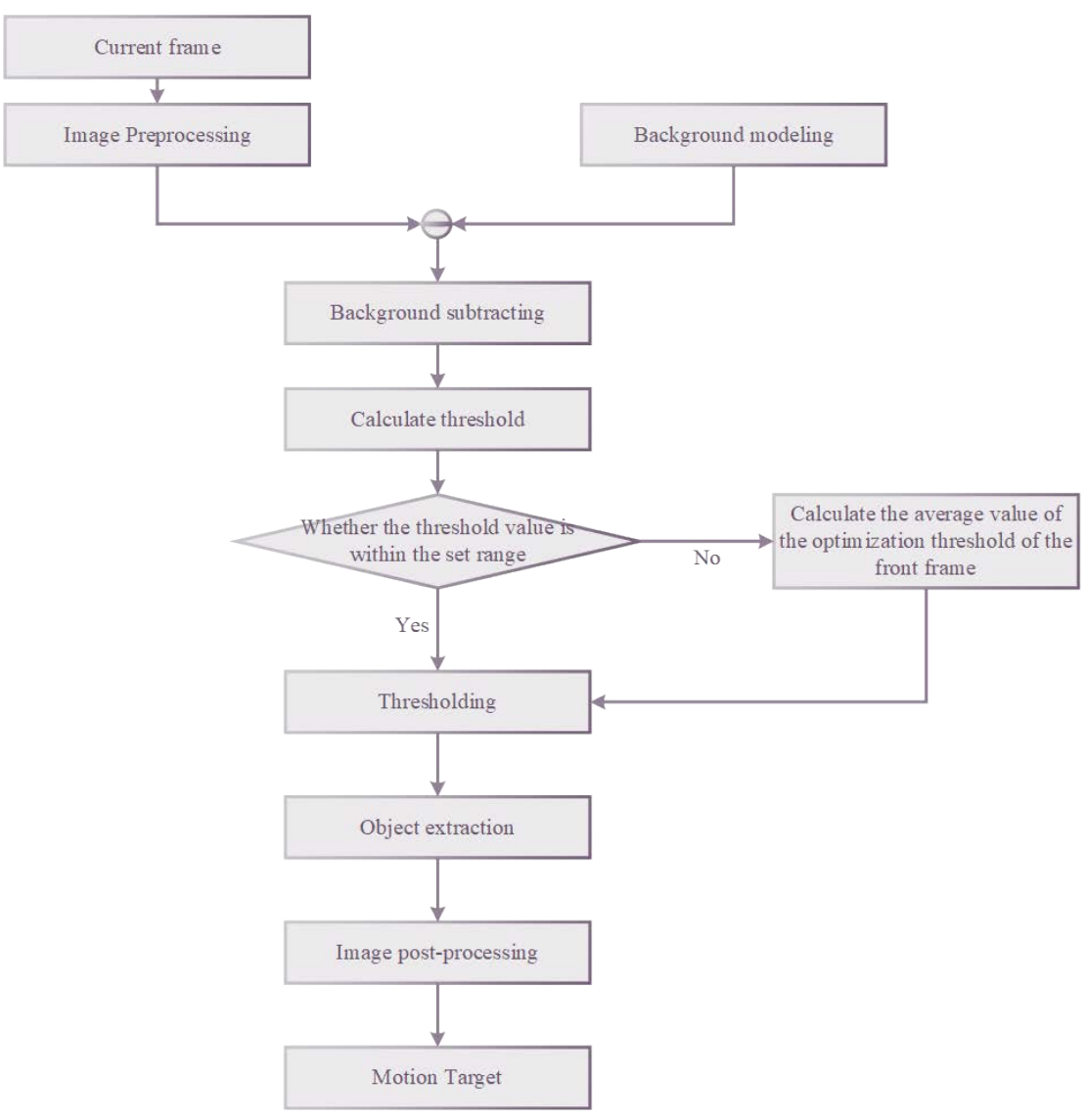

Fig.1 The flow chart of object detection

Among them, the process of background modeling and threshold selection of the binarization process [4] should be established according to different images, which is also a difficult problem in processing.

We adopt the idea of the shortest Euclidean distance in the space of the center of mass of the neighboring frame, and then classify the relations of the center of mass of different objects[5], to reach the relations of the center of mass of the moving objects.

\subsection{Centroid position and calculation}

The bitmap has specific pixels. For the representation of coordinates, let's take the upper-left coordinate of the image as $(0,0)$, and the coordinates of other positions as the basis. For every 1 pixel increase, the corresponding coordinate value is added 1 . The coordinates of the center of mass are expressed as $\left(G_{x}, G_{y}\right)$. For each goal, we use the polygon centroid formula to calculate the centroid of the area.

\section{Establishment of the volume model and approximate calculation}

If the contour of the silica area can be determined, and the appropriate contour segmentation method can be further found, the problem can be solved.

\subsection{Determine the outline of the silica area}

We use the method of 8-connected to determine the pixel region connected to the center of mass coordinate $\left(G_{x}, G_{y}\right)[6]$.

First, establish a queue q, and the centroid coordinates $\left(G_{x}, G_{y}\right)$ are added to the queue; Then extend the whole binary image pixel coordinate matrix $G_{b}$ from the center of mass coordinates $\left(G_{x}, G_{y}\right)$. 
The coordinates in the binary image pixel coordinate matrix $G_{b}$ where the pixel position point value is equal to the value at the center of mass coordinate $\left(G_{x}, G_{y}\right)$ are added to the queue q.

The new coordinate point pops up from the queue, iterates, and repeats the process a. Exit when the number of elements in the queue is empty. Traverse the pixel points of the whole image, change the coordinate point value of pixel value coordinate point value of 3 to 1 , and set the other coordinates to 0 .

\subsection{Find the suitable contour segmentation method}

After segmentation, each part is regarded as a neat sphere, and the number of white pixel points is obtained by traversing the region, that is, the pixel area. The area is regarded as a circle area, and the corresponding radius can be figured out, that is, the radius of the ball. The cutting criteria can be defined as:

(1)The aspect ratio of the cut area should be as close as possible to 1, that is, the length and width should be equal(Here the length means the difference between the $\mathrm{x}$ coordinates of the two pixels at the end of the horizontal direction, denoting $\mathrm{l}$, and the width means the difference between the $\mathrm{y}$ coordinates of the two pixels at the end of the vertical direction, denoting $w$ )

(2)The area ratio between the silica area and the non-silica area should not exceed a certain threshold.

Based on the above two principles, we apply the following process for cutting Fig.2 ( $\pi / 4$ is the ratio of the area of a circle to the total area when the largest circle is drawn in the center of a standard square. ) :

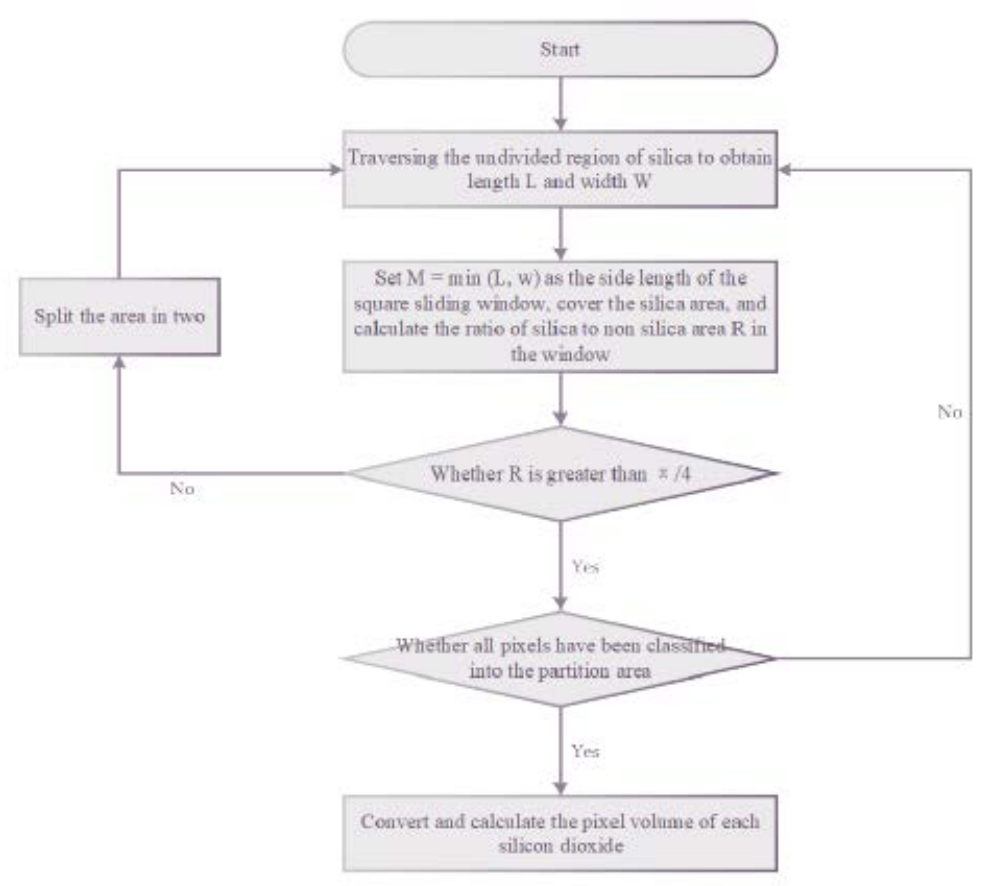

Fig.2 Flow chart of image cutting

\section{The solution of pixel volume to actual melting speed}

For any graph, it will eventually be divided into n parts. All binary image matrices are added together to obtain the pixel area $S_{x_{i}}$. The pixel radius of the region $R_{x_{i}}$ can be obtained by $S=\pi R^{2}$, and the volume of the region can be further approximated as the volume of the sphere constituted by this radius $V_{x_{i}}=(4 / 3) \pi R^{3}$, Thus, the pixel volume of the graph can be expressed as $V_{x}=\sum V_{x_{i}}$. 
The segmentation process is realized in the Matlab program according to the idea of model establishment, and obtain the pixel volume.

In the image , $1 m m=n d p, \quad 1 m^{3}=n^{3} d p^{3}$.Take the density of silicon dioxide $\rho=2.2 \times 10^{-3} \mathrm{~g} / \mathrm{mm}^{3}, V=V_{x} / \mathrm{n}^{3}, \quad m=\rho V$

The instantaneous mass change rate at the time I require only the derivative of $m_{i}$ concerning $\mathrm{t}$, $v=\Delta m_{i} / \Delta t$.

As can be seen from the figure, the melting rate was the fastest at the beginning, and the changing trend gradually decreased with time was similar to the exponential change, which was obtained by fitting the curve with the exponential form:

$v=-1.2 \times 10^{13} e^{-0.06 x}$

Is the estimated real-time melting rate of silica, As shown in Fig.3

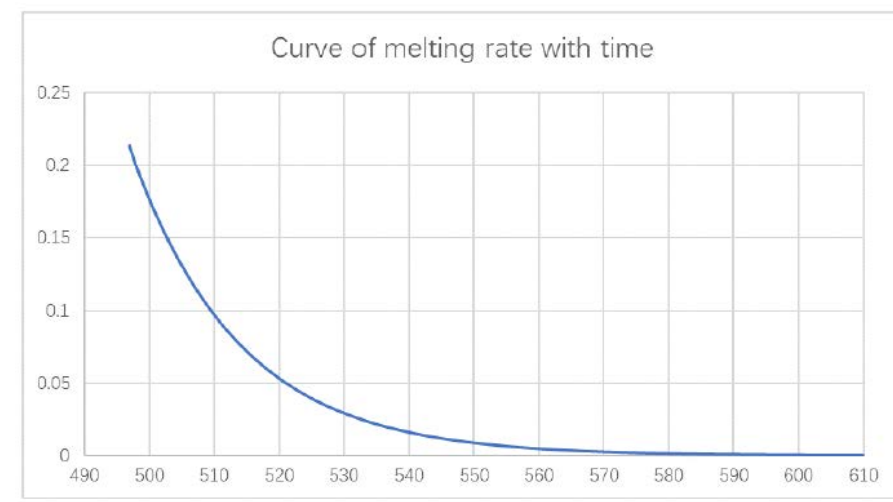

Fig.3 Curve of melting rate with time

\section{Conclusion}

For the time-series image of crystal in the molten pool, the extraction of the target crystal area is a difficult problem. In this paper, the background difference method and the centroid correlation are used to achieve better results, which can provide a better way of tracking the moving position of molten crystal. In the calculation of the final melting speed, this paper uses the idea of the microelement method and image segmentation and establishes the pixel volume method. The calculation is simple and rapid, but there are some errors. In the future work, for the estimation of the actual crystal volume, we will introduce more factors to restore the image, such as the texture and light shadow features of the image, to achieve better accuracy; at the same time, we will consider introducing more artificial intelligence algorithms to improve the detection efficiency.

\section{References}

[1] S.-h. ZHANG, X.-X. XUE, and Z.-f. JIN, "Current situation and comprehensive utilization of iron ore tailings resources in our country [j]," Journal of Materials and Metallurgy, vol. 4, no.001, 2004.

[2] O. Barnich and M. Van Droogenbroeck, "Vibe: A universal background subtraction algorithm for video sequences,” IEEE Transactions on Image processing, vol. 20, no. 6, pp. 1709-1724,2010.

[3] S. Zhi-hai, K. Wan-Zeng, and Z. Shan-an, "Moving object location in video sequences based on subtractive clustering algorithm,” Opto-Electronic Engineering, vol. 7, 2008.

[4] Zhongzeang T. Moving Object Automatic Detection, Extraction, And Tracking [D]. Anhui University.

[5] HUANG Zhan Hua, ZHAN Hao, CAI Huai yu. Researches on Real-time Detecting Track of Moving Objects of Video[J]. Journal of Optoelectronics Laser, 2002, 13(3):277-281. 
[6] Gonzalez, Rafael C, Woods, Richard E. Digital Image Processing (3rd Edition)[M]. Prentice-Hall, Inc. 2007. 